\title{
SU(4) spin-orbital two-leg ladder, square, and triangle lattices
}

\begin{abstract}
Shun-Qing Shen*
Department of Physics, The University of Hong Kong, Pokfulam Road, Hong Kong, China

(Received 29 April 2002; revised manuscript received 16 September 2002; published 19 December 2002)

Based on the generalized valence bond picture, a Schwinger boson mean-field theory is applied to the symmetric SU(4) spin-orbital systems. For a two-leg SU(4) ladder, the ground state is a spin-orbital liquid with a finite energy gap, in good agreement with recent numerical calculations. In two-dimensional square and triangle lattices, the SU(4) Schwinger bosons condense at $(\pi / 2, \pi / 2)$ and $(\pi / 3, \pi / 3)$, respectively. Spin, orbital, and coupled spin-orbital static susceptibilities become singular at the wave vectors; the Bose condensation arises at twice this value. It is also demonstrated that there are spin, orbital, and coupled spin-orbital long-range orderings in the ground state.
\end{abstract}

DOI: 10.1103/PhysRevB.66.214516

PACS number(s): 75.10.Jm, 75.40.Mg

Recently the properties of spin systems with orbital degeneracy are attracting a lot of attention. ${ }^{1}$ Several spinorbital models are proposed in various kinds of materials, such as $\mathrm{C}_{60},{ }^{2} \mathrm{NiLiO}_{3},{ }^{3} \mathrm{Na}_{2} \mathrm{Ti}_{2} \mathrm{Sb}_{2} \mathrm{O},{ }^{4}$ and $\mathrm{LaMnO}_{3} .{ }^{5}$ The interplay of spin and orbital degrees of freedom produces not only new magnetic structure phases but also novel quantum ordered and disordered states such as the orbital density wave and spin-orbital liquids. A simplified and symmetric model for these systems is ${ }^{6,7}$

$$
H=\frac{1}{2} \sum_{i, \boldsymbol{\delta}} J_{\boldsymbol{\delta}}\left(2 \mathbf{S}_{i} \cdot \mathbf{S}_{i+\boldsymbol{\delta}}+1 / 2\right)\left(2 \mathbf{T}_{i} \cdot \mathbf{T}_{i+\boldsymbol{\delta}}+1 / 2\right),
$$

where the operators $\mathbf{S}$ and $\mathbf{T}$ are $\mathbf{S U}(2)$ Pauli matrices for the spin and orbital degrees of freedom, respectively. The vector $\delta$ points to the nearest-neighboring sites. It is already known that the model possesses SU(4) symmetry, and can be derived from a quarter-filled electronic model with twofold orbital degeneracy by ignoring the Hund's rule couplings and considering the large on-site Coulomb interaction. High symmetry in this spin-orbital model means strong correlations between spins and orbitals. In an SU(4) symmetric state, the correlation functions for fifteen generators of the SU(4) Lie group are isotropic. The spin, orbital, and coupled spinorbital degrees of freedom must be treated on equal footing. ${ }^{3,8}$ Over the last few years this model was studied extensively. In one dimension (1D), it is fairly understood analytically and numerically. ${ }^{9-13}$ The one-dimensional model can be solved by means of the Bethe ansatz, and its ground state is described by a gapless spin liquid, similar to the SU(2) Heisenberg model. In 2D, it is relatively less understood. Li et ${ }^{3}{ }^{3}$ first argued that an SU(4) singlet plaquette state contains at least four sites, and a collection of such SU(4) singlets may lead to a spin liquid state. This picture is realized very well in the two-leg ladder model, ${ }^{14}$ and some solvable models. ${ }^{15}$ So far it is not clear whether such an idea can be realized in 2D. Except numerical diagonalization on small clusters ${ }^{16}$ and series expansion, ${ }^{17}$ there have been no solid results as whether the ground state is long-range ordered or a spin-orbital liquid.

In this paper, the properties of the generalized valence bond state consisting of the SU(4) singlets are discussed in detail for the model [Eq.(1)] in a SU(4) Schwinger boson mean-field theory. The ground state of the system can be either ordered or disordered, depending on the dimensionality and lattice topological structure. In a two-leg ladder system, we find that the ground state is a spin liquid state with a finite energy gap, which is in good agreement with recent numerical calculations by van den Bossche. ${ }^{14}$ In square and triangle lattices, the Schwinger bosons condense at zero temperatures, i.e., the Bose-Einstein condensation (BEC) occurs and is identified as the indication of long-range order (LRO) in the ground state. The relation between BEC and LRO is illustrated explicitly in spin, orbital, and spin-orbital static transverse susceptibilities, which become singular at the wave vectors $(\pi / 2, \pi / 2)$ and $(\pi / 3, \pi / 3)$ for the respective square and triangle lattices, leading to finite staggered magnetizations for the spin, orbital, and coupled spin-orbital densities in the thermodynamic limit. Thus, three Goldstone modes are found. According to the calculated susceptibilities, the spin, orbital, and spin-orbital LROs may coexist, but the direction of spontaneous symmetry breaking will determine the properties of the ground state.

In general, for the spin-1/2 system with double orbital degeneracy, there are four local states on each site $i$ according to the eigenvalues of $\mathbf{S}_{i}^{z}$ and $\tau_{i}^{z}:|1\rangle=|+1 / 2,+1 / 2\rangle$, $|2\rangle=|-1 / 2,+1 / 2\rangle,|3\rangle=|+1 / 2,-1 / 2\rangle,|4\rangle=|-1 / 2,-1 / 2\rangle$. Four Schwinger bosons can be introduced to describe these four states: $|\mu\rangle=a_{\mu}^{\dagger}|0\rangle$, where $|0\rangle$ is the vacuum states and $\mu=1,2,3,4$. There has to be a local constraint imposed, $\sum_{\mu=1}^{4} a_{i \mu}^{\dagger} a_{i \mu}=1$ on each lattice site. The permutation operator $P_{i j}=\left(2 \mathbf{S}_{i} \cdot \mathbf{S}_{j}+\frac{1}{2}\right)\left(2 \mathbf{T}_{i} \cdot \mathbf{T}_{j}+\frac{1}{2}\right)$ is to exchange the two states on the sites $i$ and $j, P_{i j}|i \mu, j \nu\rangle=|i \nu, j \mu\rangle$. Moreover, $P_{i j}$ can be expressed in terms of the four hard-core bosons as $P_{i j}=\Sigma_{\mu, \nu} a_{i \mu}^{\dagger} a_{i \nu} a_{j \nu}^{\dagger} a_{j \mu}$. An SU(4) singlet is defined by $\mathrm{SU}_{4}(i, j, k, l)=\Sigma_{\mu, \nu, \gamma, \delta} \Gamma_{\mu, \nu, \gamma, \delta} \quad a_{i \mu}^{\dagger} a_{j \nu}^{\dagger} a_{k \gamma}^{\dagger} a_{l \delta}^{\dagger}|0\rangle$, where $\Gamma_{\mu, \nu, \gamma, \delta}$ is an antisymmetric tensor. When the model Hamiltonian Eq. (1) has only four lattice sites, an SU(4) singlet is always the lowest-energy state for $J_{\delta} \geqslant 0$. According to the group theory, the $\mathrm{SU}(4)$ symmetric state for a lattice with $4 n$ sites ( $n$ is integer) can be regarded as a linear combination of all states consisting of $n \mathrm{SU}(4)$ singlets. ${ }^{15}$ This is a generalization of Anderson's resonating valence bond (VB) state ${ }^{18}$ from the spin $\mathrm{SU}(2)$ system to the $\mathrm{SU}(4)$ system. As is well known in the Heisenberg model, a short-range VB state may 
describe a spin liquid state with a finite energy gap, ${ }^{19}$ and a long-range VB state may possess antiferromagnetic LRO. ${ }^{20}$ A Schwinger boson mean-field theory based on the shortrange VB state was proposed for the spin $\mathrm{SU}(2)$ systems by Auerbach and Arovas, ${ }^{21}$ which successfully describes either ordered or disordered quantum states. Very recently, the theory was applied to the spin-orbital systems by the present author and his collaborator. ${ }^{8}$

To realize the generalized VB state in the SU(4) spinorbital system, the model Hamiltonian is rewritten as

$$
\begin{aligned}
H= & -\frac{1}{4} \sum_{i, \delta, \mu, \nu} J_{\delta} A_{i, i+\delta ; \mu, \nu}^{\dagger} A_{i, i+\delta ; \mu, \nu} \\
& +\sum_{i} \lambda_{i}\left(\sum_{\mu=1}^{4} a_{i, \mu}^{\dagger} a_{i, \mu}-1\right)+\frac{1}{2} N_{\Lambda} \sum_{\delta} J_{\delta},
\end{aligned}
$$

where $A_{i, j ; \mu, \nu}=a_{i \mu} a_{j \nu}-a_{i \nu} a_{j \mu}$ and $N_{\Lambda}$ is the total number of lattice sites. Antisymmetric operators $A_{i, j ; \mu, \nu}$ are introduced for the purpose of the mean-field calculations. The following theory is limited to the case $J_{\delta} \geqslant 0$. The local Lagrangian multiplier is also used to impose the local constraint for the hard-core bosons on average. In the mean-field approximation we will take all $\lambda_{i}=\lambda$. The thermodynamic averages of the operators $A_{i j, \mu \nu}$ are defined as the VB order parameters $\left\langle A_{i, i+\delta ; \mu, \nu}\right\rangle \equiv-2 i \Delta_{\mu, \nu}(\delta)$, which are odd functions with respect to either the indices $\mu, \nu$ or the vector direction $\delta$. In the momentum space, we define

$$
\gamma_{\mu, \nu}(\mathbf{k}) \equiv 2 i \sum_{\delta} J_{\delta} \Delta_{\mu \nu}(\delta) \exp (i \mathbf{k} \cdot \delta)
$$

We define an eight-component spinor

$$
\Phi_{\mathbf{k}}^{\dagger}=\left(a_{\mathbf{k} 1}^{\dagger}, a_{\mathbf{k} 2}^{\dagger}, a_{\mathbf{k} 3}^{\dagger}, a_{\mathbf{k} 4}^{\dagger}, a_{-\mathbf{k} 1}, a_{-\mathbf{k} 2}, a_{-\mathbf{k} 3}, a_{-\mathbf{k} 4}\right) .
$$

By utilizing the Pauli matrices $\sigma_{\alpha}(\alpha=x, y, z)$, the decoupled mean-field Hamiltonian is thus written in a compact matrix form,

$$
H=\frac{1}{2} \sum_{\mathbf{k}} \Phi_{\mathbf{k}}^{\dagger}\left[\lambda-i \sigma_{y} \otimes \mathbf{B}(\mathbf{k})\right] \Phi_{\mathbf{k}}+\mathcal{E}_{0},
$$

where $\mathcal{E}_{0} / N_{\Lambda}=\Sigma_{\delta} J_{\delta} \Delta_{\mu \nu}^{2}(\delta)-3 \lambda+\frac{1}{2} \sum_{\delta} J_{\delta}$;

$$
\mathbf{B}(\mathbf{k})=\left(\begin{array}{cccc}
0 & \gamma_{12}(\mathbf{k}) & \gamma_{13}(\mathbf{k}) & \gamma_{14}(\mathbf{k}) \\
-\gamma_{12}(\mathbf{k}) & 0 & \gamma_{23}(\mathbf{k}) & \gamma_{24}(\mathbf{k}) \\
-\gamma_{13}(\mathbf{k}) & -\gamma_{23}(\mathbf{k}) & 0 & \gamma_{34}(\mathbf{k}) \\
-\gamma_{14}(\mathbf{k}) & -\gamma_{24}(\mathbf{k}) & -\gamma_{34}(\mathbf{k}) & 0
\end{array}\right),
$$

where $N_{\Lambda}$ is the number of lattice sites. Considering the symmetry in the Hamiltonian, ${ }^{22}$ there exist two sets of solutions: (i) $\quad \gamma_{12}(\mathbf{k})=\gamma_{34}(\mathbf{k}), \quad \gamma_{13}(\mathbf{k})=-\gamma_{24}(\mathbf{k}), \quad \gamma_{14}(\mathbf{k})$ $=\gamma_{23}(\mathbf{k}) ; \quad$ (ii) $\quad \gamma_{12}(\mathbf{k})=-\gamma_{34}(\mathbf{k}), \quad \gamma_{13}(\mathbf{k})=\gamma_{24}(\mathbf{k}), \quad \gamma_{14}(\mathbf{k})$ $=-\gamma_{23}(\mathbf{k})$. The physical reason is that an SU(4) singlet plaquette state $\mathrm{SU}_{4}(i, j, k, l)$ contains four creation operators with different sites and indices, and $A_{i j, \mu \nu}^{\dagger}$ contains only two sites and two indices. To form such an SU(4) singlet plaquette state, the four creation operators on different sites should have different indices. The relations in solutions (i) and (ii) reflect these properties. In each $\mathrm{SU}(4)$ singlet plaquette it consists of two configurations. Each configuration consists of two spin singets and two orbital singlets. The two configurations are degenerate, but not orthogonal. The double degeneracy of the solutions may be related to the properties of SU(4) singlet plaquettes. So these relations will help us to construct the wave function of the generalized SU(4) VB state. We first focus on solution (i), and will discuss the results of solution (ii). The single-particle Green function is given by

$$
\begin{aligned}
G\left(\mathbf{k}, i \omega_{n}\right) & \equiv\left[i \omega_{n} \Omega_{1}-\lambda+i \sigma_{y} \otimes \mathbf{B}(\mathbf{k})\right]^{-1} \\
& =\frac{i \omega_{n} \Omega_{1}+\lambda+\gamma_{12}(\mathbf{k}) \Omega_{2}+\gamma_{13}(\mathbf{k}) \Omega_{3}+\gamma_{13}(\mathbf{k}) \Omega_{4}}{\left(i \omega_{n}\right)^{2}-\omega(\mathbf{k})^{2}},
\end{aligned}
$$

where $\Omega_{1}=\sigma_{z} \otimes \sigma_{0} \otimes \sigma_{0}, \quad \Omega_{2}=\sigma_{y} \otimes \sigma_{0} \otimes \sigma_{y}, \quad \Omega_{3}=\sigma_{y} \otimes \sigma_{y}$ $\otimes \sigma_{z}$, and $\Omega_{4}=\sigma_{y} \otimes \sigma_{y} \otimes \sigma_{x}$. There is only one fourfold degenerate quasiparticle spectrum,

$$
\omega(\mathbf{k})=\sqrt{\lambda^{2}-\left[\gamma_{12}^{2}(\mathbf{k})+\gamma_{13}^{2}(\mathbf{k})+\gamma_{14}^{2}(\mathbf{k})\right]},
$$

from which the free energy for the system is evaluated,

$$
F=\frac{4}{\beta} \sum_{\mathbf{k}} \ln [1-\exp [-\beta \omega(\mathbf{k})]]+2 \sum_{\mathbf{k}} \omega(\mathbf{k})+\mathcal{E}_{0} .
$$

The saddle-point equations are thus derived by minimizing the free energy with respect to the mean-field variables $\lambda$ and $\Delta_{\mu \nu}(\delta)$. We can deduce the VB order parameters according to the symmetry of $\Delta_{\mu \nu}(\delta)$ and of the lattice.

Now we apply the general formalism to the SU(4) model on several lattices. We first study the two-leg ladder model. Recent numerical study has shown that its ground state is a spin-orbital liquid with a finite energy gap, ${ }^{14}$ which can be regarded as a realization of the SU(4) plaquette state, or a short-range generalized VB state. Here the isotropic case is considered to be $J_{\|}=J_{\perp}=J$ so that we introduce two sets of VB order parameters: $\Delta_{\mu \nu}(x)$ along the ladder and $\Delta_{\mu \nu}(y)$ along the rungs. Special attention should be paid for the direction along the rung. The momentum along the rung has two discrete values. The spectra are given by

$$
\omega_{ \pm}(\mathbf{k})=\sqrt{\lambda^{2}-16 J^{2} \Delta_{\|}^{2}(\sin \mathbf{k} \pm \eta)^{2}}
$$

where $\quad \Delta_{\|}^{2}=\Delta_{12}^{2}(x)+\Delta_{13}^{2}(x)+\Delta_{14}^{2}(x), \quad$ and $\eta$ $=\Delta_{\mu \nu}(y) / 2 \Delta_{\mu \nu}(x)$ is determined by the mean field equations. $^{23}$ The two branches of the spectra have a relation: $\omega_{+}(\mathbf{k})=\omega_{-}(-\mathbf{k})$. The saddle-point equations are determined by minimizing the free energy with respect to the mean-field variables

$$
\begin{gathered}
\int \frac{d \mathbf{k}}{2 \pi} \frac{\lambda}{\omega_{+}(\mathbf{k})}\left[2 n_{B}\left(\omega_{+}(\mathbf{k})\right)+1\right]=\frac{3}{2}, \\
\int \frac{d \mathbf{k}}{2 \pi} \frac{(\sin \mathbf{k}+\eta)^{2}}{\omega_{+}(\mathbf{k})}\left[2 n_{B}\left(\omega_{+}(\mathbf{k})\right)+1\right]=\frac{1+2 \eta^{2}}{2 J},
\end{gathered}
$$




$$
\int \frac{d \mathbf{k}}{2 \pi} \frac{\sin \mathbf{k}+\eta}{\omega_{+}(\mathbf{k})}\left[2 n_{B}\left(\omega_{+}(\mathbf{k})\right)+1\right]=\frac{\eta}{J},
$$

where $n_{B}(x)$ is the distribution function for bosons: $n_{B}(x)$ $=1 /[\exp (\beta x)-1]$ and $\beta=1 / k_{B} T$. At $T=0, n_{B}\left(\omega_{+}(\mathbf{k})\right)=0$ when $\omega_{+}(\mathbf{k}) \neq 0$. The numerical calculations give rise to $\lambda$ $=2.26574 J, \Delta_{\|}=0.35339$, and $\eta=0.55146$. The minima of the spectra is at $\mathbf{k}^{*}=\pi / 2$ for $\omega_{+}(\mathbf{k})$ and at $-\pi / 2$ for $\omega_{-}(\mathbf{k}): \min \left(\omega_{ \pm}(\mathbf{k})\right)=\sqrt{\lambda^{2}-16 J^{2} \Delta_{\|}^{2}(1+\eta)^{2}}$. From the dynamic susceptibilities of spin, orbital, and spin-orbital operators, we find that there is a finite energy gap: $\Delta_{\text {gap }}$ $=2 \min \left(\omega_{ \pm}(\mathbf{k})\right)=1.138 \mathrm{~J}$. For the second set of solutions (ii), it also produces the same numerical results, degenerated with the first set of solutions. van den Bossche et al. ${ }^{14}$ studied this SU(4) ladder model up to 16 sites by an exact diagonalization method, and a finite energy gap has been found in a singlet-multiplet excitation: $\Delta=1.09 \mathrm{~J}$. The two values are in excellent agreement. A local minimum at $\mathbf{k}=\pi / 2$ in the quasiparticle dispersion was also observed. Moreover, the ground state has also been found to have a twofold degeneracy in the thermodynamic limit, consistent with the two solutions of ours. All these facts can be regarded as strong support for our present theory. It is worth noting that the theory may fail for a one-dimensional chain to predict a small energy gap due to the ignorance of the topological terms or "Pontryagin index," which can destroy Haldane's gap. The same problem was encountered in the SU(2) theory, and was discussed extensively in one-dimensional spin-1/2 systems. $^{21}$

Next we come to study two-dimensional lattices. Let us consider a square lattice first. In this case, we still assume isotropic couplings $J_{x}=J_{y}$. The spectrum for the bosonic quasiparticles can be written as 24

$$
\omega(\mathbf{k})=\sqrt{\lambda^{2}-16 J^{2} \Delta^{2}\left(\sin \mathbf{k}_{x}+\sin \mathbf{k}_{y}\right)^{2}},
$$

with $\Delta^{2}=\Delta_{12}^{2}+\Delta_{13}^{2}+\Delta_{14}^{2}$. The minimum of the energy spectra occurs at $\mathbf{k}^{*}=(\pi / 2, \pi / 2)$. The saddle-point equations are given by

$$
\begin{gathered}
\int \frac{d \mathbf{k}}{(2 \pi)^{2}} \frac{\lambda}{\omega(\mathbf{k})}\left[2 n_{B}(\omega(\mathbf{k}))+1\right]=\frac{3}{2}, \\
\int \frac{d \mathbf{k}}{(2 \pi)^{2}} \frac{\left(\sin \mathbf{k}_{x}+\sin \mathbf{k}_{y}\right)^{2}}{\omega(\mathbf{k})}\left[2 n_{B}(\omega(\mathbf{k}))+1\right]=\frac{z}{4 J},
\end{gathered}
$$

where the coordinate number for square lattice is $z=4$. In the present theory the number of bosons in the diagonalized Hamiltonian is not equal to the number of the hard-core bosons since the Bogoliubov transformation changes the number of bosons. The quasiparticle number is determined by solutions of the saddle-point equations self-consistently. At $T=0$ the bosons may condense, i.e., the BEC occurs. The minima of the boson spectra $\omega(\mathbf{k})$ are at $\mathbf{k}= \pm \mathbf{k}^{*}$. Since the distribution function becomes singular at $\omega\left(\mathbf{k}^{*}\right)=0$ in Eq. (10a), we have to introduce a finite quantity $b_{0}$ $=2\left[n_{B}\left(\omega\left(\mathbf{k}^{*}\right)\right)+n_{B}\left(\omega\left(-\mathbf{k}^{*}\right)\right)\right] /\left[N_{\Lambda} \omega\left(\mathbf{k}^{*}\right)\right]$ such that the saddle-point equations have physical solutions. The nonzero $b_{0}$ may be used as the order parameters for long-range correlations for the spin, orbital, and coupled spin-orbital densities. The saddle-point equations are solved numerically. When $T>0, b_{0}=0$. At $T=0$, we have $\lambda=8 \mathrm{~J} \Delta$ $=3.05905 \mathrm{~J}$ and $b_{0}=0.1068$. The nonzero $b_{0}$ indicates that the BEC occurs on a square lattice.

Another typical two-dimensional lattice is the triangle lattice. For example, $\mathrm{NiLiO}_{3}$ has a $2 \mathrm{D}$ triangle lattice structure, and was modeled as a spin-orbital system. ${ }^{3,25}$ Usually the quantum frustration is anticipated to make quantum fluctuations more stronger. Topologically, we can distort a triangle lattice into a square one by introducing a finite diagonal coupling $J_{x+y}$, and take another diagonal coupling $J_{x-y}=0$. In the present theory we have three sets of order parameters: $\Delta_{\mu \nu}(\mathbf{x}), \Delta_{\mu \nu}(\mathbf{y})$, and $\Delta_{\mu \nu}(\mathbf{x}+\mathbf{y})$. We focus on the isotropic case $J_{x}=J_{y}=J_{x+y}$; then the VB order parameters have a relation $\Delta_{\mu \nu}(\mathbf{x})=\Delta_{\mu \nu}(\mathbf{y})=\Delta_{\mu \nu}(\mathbf{x}+\mathbf{y})=\Delta_{\mu \nu}$. The spectra for the bosonic quasiparticles is thus given by

$$
\omega(\mathbf{k})=\sqrt{\lambda^{2}-16 J^{2} \Delta^{2}\left[\sin \mathbf{k}_{x}+\sin \mathbf{k}_{y}+\sin \left(\mathbf{k}_{x}+\mathbf{k}_{y}\right)\right]^{2}},
$$

with $\Delta^{2}=\Delta_{12}^{2}+\Delta_{13}^{2}+\Delta_{14}^{2}$. The minimum of the spectra occurs at $\mathbf{k}^{*}= \pm(\pi / 3, \pi / 3)$, which can be shifted away if the couplings are anisotropic. A similar set of saddle-point equations are obtained by minimizing the free energy in Eq. (6). Numerically solving the self-consistent equation at $T=0$ gives rise to $\lambda=6 \sqrt{3} J \Delta=3.57878 J$ and $b_{0}=0.155345$. Since $\omega\left(\mathbf{k}^{*}\right)=0$, the BEC also appears on an isotropic triangle lattice. The coupling $J_{x+y}$ does not enhance the quantum frustration to suppress the BEC completely. The role of $J_{x+y}$ is to force the minimal point from $\pm(\pi / 2, \pi / 2)$ for $J_{x+y}=0$ to $\pm(\pi / 3, \pi / 3)$ for $J_{x+y}=J_{x}=J_{y}$. A more detailed calculation shows that the wave vector $\mathbf{k}^{*}$ changes continuously as a function of $J_{x+y} / J_{x}$.

In the usual Schwinger boson mean-field theory the BEC is identified as the long-range correlations between the $\mathrm{SU}(2)$ spins. To establish the relation between the BEC and the long-range order in the present SU(4) ground state, we calculated the static susceptibilities ${ }^{26}$

$$
\begin{aligned}
\chi_{X}(\mathbf{q})= & -\frac{1}{16 N_{\Lambda}} \sum_{\mathbf{k}} \times \operatorname{Tr}\left\{\Omega_{X} G\left(\mathbf{k}+\mathbf{q}, \tau=0^{-}\right)\right. \\
& \left.\times \Omega_{X} G\left(\mathbf{k}, \tau=0^{+}\right)\right\},
\end{aligned}
$$

where $X=S$ for spin $\mathbf{S}_{i}^{z}, X=T$ for orbital $\mathbf{T}_{i}^{z}$, and $X=S T$ for the coupled spin-orbital $2 \mathbf{S}_{i}^{z} \mathbf{T}_{i}^{z}$. These three operators can be expressed in terms of spinors, $\Phi_{i}^{\dagger} \Omega_{X} \Phi_{i} / 4$, where $\Omega_{S}=\sigma_{0}$ $\otimes \sigma_{0} \otimes \sigma_{z}, \Omega_{T}=\sigma_{0} \otimes \sigma_{z} \otimes \sigma_{0}$, and $\Omega_{S T}=\sigma_{0} \otimes \sigma_{z} \otimes \sigma_{z}$. From the single-particle Green function Eq. (4), we can calculate the static susceptibilities

$$
\begin{aligned}
& \chi_{S}(\mathbf{Q}) / N_{\Lambda} \approx \frac{1}{4} b_{0}^{2} \gamma_{13}^{2}\left(\mathbf{q}=2 \mathbf{k}^{*}\right), \\
& \chi_{T}(\mathbf{Q}) / N_{\Lambda} \approx \frac{1}{4} b_{0}^{2} \gamma_{12}^{2}\left(\mathbf{q}=2 \mathbf{k}^{*}\right), \\
& \chi_{S T}(\mathbf{Q}) / N_{\Lambda} \approx \frac{1}{4} b_{0}^{2} \gamma_{14}^{2}\left(\mathbf{q}=2 \mathbf{k}^{*}\right),
\end{aligned}
$$


which become singular when $\mathbf{q}=\mathbf{Q}=2 \mathbf{k}^{*}$ and higher-order terms can be ignored. The correlation functions are proportional to the number of lattice sites once $b_{0} \neq 0$. These properties are characterized by long-range correlations at the wave vector $\mathbf{Q}$. In the thermodynamic limit, the corresponding magnetizations become $m_{X}=\sqrt{\chi_{X}(Q) / N_{\Lambda}}$, which depend on the values of VB order parameters $\Delta_{12}^{2}, \Delta_{13}^{2}$, and $\Delta_{14}^{2}$, respectively. The long-range order is thus at $\mathbf{Q}$ $=(\pi, \pi)$ for a square lattice $[(\pi, \pi)$ and $(-\pi,-\pi)$ are the same vector], and $\mathbf{Q}=(2 \pi / 3,2 \pi / 3)$ or $(-2 \pi / 3,-2 \pi / 3)$ for a triangle lattice. The two vectors $\pm k^{*}$ correspond to one state for a square lattice, but two equivalent states for a triangle lattice. From the second set of mean field solutions, we find that the above relations remain if we make a permutation between $\mathbf{S}$ and $\mathbf{T}$. Therefore these two solutions (i) and (ii) are degenerate. We believe that the double degeneracy of the ground states observed in our theory is not a result of the mean-field theory, and may have a deep physical origin. The singularity in static susceptibilities also reflects the fact that the collective modes are gapless Goldstone modes. The SU(4) system may have at most three Goldstone mode when the symmetry is broken spontaneously.

When $\Delta_{12}^{2}=\Delta_{13}^{2}=\Delta_{14}^{2}=\Delta^{2} / 3$, we have $\chi_{S}=\chi_{T}=\chi_{S T}$. These relations are in agreement with the isotropic correlations for the spin, orbital, and coupled spin orbital in our SU(4) invariant ground state. In such a mean-field theory, the operator $\mathbf{S}^{z}, \mathbf{T}^{z}$, and $2 \mathbf{S}^{z} \mathbf{T}^{z}$ play an equal role. When spin and orbital density operators have long-range correlations, and the coupled spin-orbital density operators also have long-range correlations with the same wave vectors. An interesting observation is that the ground-state energy depends only on the parameter $\Delta^{2} .{ }^{27}$ It may contain some new states, which is determined by the direction of spontaneous symmetry breaking. ${ }^{28}$ Actually, we have the freedom to choose the direction of the spontaneous symmetry breaking in the thermodynamic limit. For example, an infinitesimal external staggered magnetic field along the spin $z$ direction may induce a ground state with $\Delta_{12}^{2}=\Delta^{2}$ and $\Delta_{13}^{2}=\Delta_{14}^{2}=0$, where only a magnetic long-range order appears. An infinitesimal Jahn-Teller distortion may induce a ground state with $\Delta_{13}^{2}$ $=\Delta^{2}$ and $\Delta_{12}^{2}=\Delta_{14}^{2}=0$, where an orbital long-range order shows up. It is also possible for two or more types of longrange orders to coexist in a single ground state.

In conclusion, based on a generalized valence bond state picture, a Schwinger boson mean-field theory is developed for the symmetric SU(4) spin-orbital systems, showing that the ground state for a two-leg ladder model is a spin-orbital liquid with a finite energy gap in low-energy excitations, and the ground states for square and triangle lattices possess spin, orbital, and coupled spin-orbital long-range orderings.

I would like to thank G. M. Zhang for his helpful discussions. This work was supported by a RGC grant from Hong Kong and a CRCG grant from The University of Hong Kong.
*Electronic address: sshen@hkucc.hku.hk

${ }^{1}$ Y. Tokura and N. Nagaosa, Science 288, 462 (2001).

${ }^{2}$ D.P. Arovas and A. Auerbach, Phys. Rev. B 52, 10114 (1995).

${ }^{3}$ Y.Q. Li, M. Ma, D.N. Shi, and F.C. Zhang, Phys. Rev. Lett. 81, 3527 (1998).

${ }^{4}$ S.K. Pati, R.R.P. Singh, and D.I. Khomskii, Phys. Rev. Lett. 81, 5406 (1998).

${ }^{5}$ S. Ishihara, J. Inoue, and S. Maekawa, Physica C 263, 130 (1997).

${ }^{6}$ I. Kugel and D.I. Khomskii, Zh. Eksp. Teor. Fiz. 64, 1429 (1973) [Sov. Phys. JETP 37, 725 (1973)].

${ }^{7}$ C. Castellani, C.R. Natoli, and J. Ranninger, Phys. Rev. B 18, 4945 (1978).

${ }^{8}$ G.M. Zhang and S.Q. Shen, Phys. Rev. Lett. 87, 157201 (2001); S.Q. Shen and G.M. Zhang, Europhys. Lett. 57, 274 (2002).

${ }^{9}$ B. Sutherland, Phys. Rev. B 12, 3795 (1975).

${ }^{10}$ P. Azaria, A.O. Gogolin, P. Lecheminant, and A.A. Nersesyan, Phys. Rev. Lett. 83, 624 (1999).

${ }^{11}$ C. Itoi, S. Qin, and I. Affleck, Phys. Rev. B 61, 6747 (2000).

${ }^{12}$ B. Frischmuth, F. Mila, and M. Troyer, Phys. Rev. Lett. 82, 835 (1999)

${ }^{13}$ W. Zheng and J. Oiitmaa, Phys. Rev. B 64, 014410 (2001).

${ }^{14}$ M. van den Bossche, P. Azaria, P. Lecheminant, and F. Mila, Phys. Rev. Lett. 86, 4124 (2001).

${ }^{15}$ S.Q. Shen, Phys. Rev. B 64, 132411 (2001).

${ }^{16}$ M. van den Bossche, F. Mila, and F.C. Zhang, Eur. Phys. J. B 17, 367 (2000).

${ }^{17}$ E. Zasinas, O.P. Sushkov, and J. Oitmaa, Phys. Rev. B 64, 184431 (2001).
${ }^{18}$ P.W. Anderson, Mater. Res. Bull. 8, 153 (1973).

${ }^{19}$ I. Affleck, T. Kennedy, E.H. Lieb, and H. Tasaki, Phys. Rev. Lett. 59, 799 (1987).

${ }^{20}$ S. Liang, B. Doucot, and P.W. Anderson, Phys. Rev. Lett. 61, 365 (1988); S. Liang, Phys. Rev. B 42, 6555 (1990).

${ }^{21}$ A. Auerbach and D.P. Arovas, Phys. Rev. Lett. 61, 617 (1988); D.P. Arovas and A. Auerbach, Phys. Rev. B 38, 316 (1988). For a review, see A. Auerbach, Interacting Electrons and Quantum Magnetism (Springer, New York, 1998).

${ }^{22}$ Diagonalization of the Hamiltonian [Eq. (3)] gives two branches of the double degenerated spectra, $\omega_{+}(k)$ and $\omega_{-}(k)$. The general expressions of $\omega_{+}(k)$ and $\omega_{-}(k)$ in terms of six order parameters $\Delta_{\mu \nu}$ are rather lengthy. It can be derived explicitly with the help of softwares such as MATHEMATICA or MATHLAB, and generally $\omega_{+}(k) \neq \omega_{-}(k)$. In this case the four types of Schwinger bosons do not play the same role in the mean-field state. Consequently, it will lead to the anisotropy of the spin, orbital, and coupled spin-orbital correlation functions, and the solutions have no SU(4) symmetry. To keep the SU(4) symmetry of the state and the isotropic correlation functions we have to place some restriction on the six order parameters. We take $\omega_{+}(k)=\omega_{-}(k)=\omega(k)$ such that the spectra become fourfold degenerate. The equality gives the two sets of the solutions.

${ }^{23} \eta$ is independent of $\mu \nu$, which can be derived from the meanfield equations if we list all the equations for $\Delta_{\mu \nu}(\delta)$.

${ }^{24}$ The spectra in Eqs. (9) and (11) have no rotation symmtries of square and triangle lattices. Under the rotation symmetry, we have several other solutions which give the same physical consequences. It is worth noting that the dispersion relations for 
Schwinger bosons are not observables, and the physical observables are related to the spin operators explicitly.

${ }^{25}$ F. Reynaud, D. Mertz, F. Celestini, J.-M. Debierre, A.M. Ghorayeb, P. Simon, A. Stepanov, J. Voiron, and C. Delmas, Phys. Rev. Lett. 86, 3638 (2001).

${ }^{26}$ J. R. Schrieffer, Theory of Superconductivity (Addison-Wesley, Reading, MA, 1984).

${ }^{27}$ S.Q. Shen and X.C. Xie, J. Phys.: Condens. Matter 8, 4805 (1996), where a similar result was observed in the SO(4) theory of the negative $U$ Hubbard model for superconductivity and the charge-density wave.

${ }^{28}$ The same physics exists in the spin SU(2) Heisenberg model. In an $\mathrm{SU}(2)$ invariant state, spin-correlation functions along different axes are isotropic. When there exists LRO in the thermodynamic limit, an infinitesimal external field is expected to induce symmetry breaking spontaneously along only one specific axis, say the $\mathrm{z}$ axis. See P. W. Anderson, Basic Notions of Condensed Matter Physics (Benjamin-Cummings, London, 1984). 